\title{
Interference Sensitivity for Multi-user MIMO in LTE
}

\author{
Rizwan Ghaffar, Raymond Knopp \\ Eurecom, 2229 route des Crêtes B.P.193 \\ 06904 Sophia Antipolis Cedex FRANCE \\ Email: rizwan.ghaffar@eurecom.fr, raymond.knopp@eurecom.fr
}

\begin{abstract}
A recent idea of exploiting the promised gains of multi-user MIMO for future cellular standards as LTE by intelligent interference aware receivers instead of suboptimal singleuser receivers is quite promising. However this detection strategy is based on the exploitation of the structure of interference which necessitates the knowledge of the constellation and precoder of the co-scheduled user equipment (UE). As the downlink control information (DCI) in LTE is devoid of this information, we look in this paper at the sensitivity of LTE's multi-user MIMO mode to the information of co-scheduled UE. We show that exploiting the discrete nature of interference in the absence of the exact knowledge of interference constellation has significant gains as compared to the suboptimal single-user detection. To this end, we propose a blind receiver for LTE which shows significantly improved performance as compared to single-user receivers and exhibits a minor degradation as compared to interference aware receivers.
\end{abstract}

Index Terms-Multiuser MIMO, LTE, Interference sensitivity, Discrete constellation, Interference exploitation

\section{INTRODUCTION}

Wireless communication has been revolutionized in the last decade by the addition of spatial dimension to the classical dimensions of time and frequency. This new dimension in the form of multiple antennas at the transmitter and receiver (MIMO) promises improved reliability, higher spectral efficiency and spatial separation of users [1]. The exploitation of these spatial degrees of freedom in the downlink of multiuser cellular systems for serving multiple users on the same time-frequency resources, i.e. multi-user MIMO or user multiplexing is particularly beneficial for achieving high data rates envisioned for future cellular standards as third generation partnership project long term evolution (3GPP LTE) [2] and LTE-Advanced [3].

The promised gains of multi-user MIMO are highly dependent on the availability of channel state information at the transmitter (CSIT) [4] however its acquisition in a practical system particularly frequency division duplex (FDD) system is far from realizable. Future wireless systems are therefore considering single-user and multi-user MIMO strategies based on limited or quantized CSIT. 3GPP LTE is focusing on precoder codebook based approach [2] which is characterized by low resolution. This low level quantization of LTE precoder codebook questions the feasibility of multi-user MIMO mode if suboptimal single-user receivers are employed by the users [5] (page 244). However significant gains can be obtained if instead of ignoring the residual interference (signal of coscheduled user) or assuming it to be Gaussian, the structure of this discrete interference is exploited by the users in the detection process by employing interference aware receivers [6]. However the prerequisites of this interference aware receiver are, the knowledge of the interference channel and its constellation. Though the predefined scheduling strategies combined with the small cardinality of LTE codebooks for multi-user MIMO mode can enable the users to find the interference channels (being the product of own channel and coscheduled user's precoder) but the information regarding the constellation of co-scheduled user is still eluded as downlink control information (DCI) formats in 3GPP LTE [7] do not allow the transmission of this information to the users.

In this paper, we look at the sensitivity of multi-user MIMO mode in LTE to the knowledge of the constellation of coscheduled user (interference) and propose a blind receiver to this effect. We show that significant performance gains can be achieved by considering the finitary restrictions of interference instead of its unrealistic Gaussian assumption, though the lack of the information regarding interference constellation slightly degrades the performance. This analysis on one hand proposes a solution of exploiting the discrete nature of interference when its constellation is not known in LTE while on the other hand, it underlines the significance of this information thereby motivating its consideration to be included in the ongoing standardization of LTE-Advanced.

Regarding notations, we will use lowercase or uppercase letters for scalars, lowercase boldface letters for vectors and uppercase boldface letters for matrices. |.| and ||.|| indicate norm of scalar and vector while $(.)^{T},(.)^{*}$ and $(.)^{\dagger}$ indicate transpose, conjugate and conjugate transpose respectively. $(.)_{R}$ and $(.)_{I}$ indicate real and imaginary parts.

The paper is divided into five sections. In section II, we give an overview of LTE where we define the system model. Section III focuses on the sensitivity of LTE multi-user MIMO mode to the information of interference where we propose a blind receiver for LTE multi-user MIMO mode. Section IV encompasses the simulation results which are followed by the conclusions. 


\section{LTE SySTEM MOdEL}

\section{A. LTE - A Brief Overview}

3GPP LTE supports maximum of four transmit and receive antennas $(4 \times 4$ system $)$, however $2 \times 2$ MIMO system is assumed as the baseline configuration for the downlink [2]. We therefore restrict ourselves to the baseline configuration in this paper with the eNodeB (LTE notation for the base station) equipped with two antennas while we consider single and dual antenna user equipments (UEs). Like other ongoing standardizations of wireless communication systems as IEEE 802.16m (WiMAX) and IEEE 802.11n (Wireless LAN), LTE is also based on OFDMA (Orthogonal Frequency Division Multiple Access) combined with bit interleaved coded modulation (BICM) [8]. The defined SISO (Single Input Single Output), SIMO (Singe-Input Multiple-Output) and MIMO transmission schemes are categorized in the following seven transmission modes.

- Transmission mode 1. Single Input Single Output (SISO).

- Transmission mode 2. Fall back transmit diversity. Transmission rank is one, i.e. one data stream for each UE. Employs Alamouti space-time or space-frequency codes [9].

- Transmission mode 3. Open loop spatial multiplexing based on cyclic delay diversity (CDD). Transmission rank is greater than 1 .

- Transmission mode 4. Closed-loop spatial multiplexing. Transmission rank is two, i.e. two codewords are transmitted to the UE in single-user MIMO mode.

- Transmission mode 5. Multi-user MIMO mode. Supports only rank one transmission, i.e. one codeword for each UE.

- Transmission mode 6. Closed-loop precoding for rank one transmission, i.e. one codeword to the UE in singleuser MIMO mode.

- Transmission mode 7. Beamforming based on direction of arrival (DoA) estimations from the uplink.

For closed loop transmission modes (mode 4, 5 and 6), precoding mechanisms are employed at the transmit side with the objective of maximizing throughput. In 3GPP LTE, different codebooks have been defined depending on the number of transmit antenna ports. For the case of eNodeB with two antennas, LTE defines following four precoders.

$$
\mathbf{p}=\frac{1}{\sqrt{2}}\left[\begin{array}{l}
1 \\
1
\end{array}\right], \frac{1}{\sqrt{2}}\left[\begin{array}{c}
1 \\
-1
\end{array}\right], \frac{1}{\sqrt{2}}\left[\begin{array}{l}
1 \\
j
\end{array}\right], \frac{1}{\sqrt{2}}\left[\begin{array}{c}
1 \\
-j
\end{array}\right]
$$

In order to avoid excessive downlink signaling, transmission mode for each UE is configured semi-statically via higher layer signaling. The precoding is selected and applied by the eNodeB to the data transmission to a target UE based on the channel feedback received from that UE. This feedback includes a channel rank indicator (RI), channel quality indicator (CQI) and precoding matrix indicator (PMI). The RI indicates the estimated number of simultaneous layers which can be received by the UE. Note that one or more layers can be mapped to the same codeword (data stream) and are jointly encoded for transmission to the same target UE. The RI is a wideband measure, i.e. the same channel rank is assumed on all allocated resources. CQI and PMI are indices to the tables, i.e. the former is an index to the table of modulation coding scheme (MCS) and transport block size (TBS) comprising 32 entries while the latter is an index to the codebook defined for a given number of transmit antenna ports $(1,2,4)$. The derivation of CQI information is based on the assumption that the selected PMI will be applied to the next scheduled transmission.

This information is fed back by the UE on PUSCH (Physical uplink shared channel) to the eNodeB. The eNodeB then transmits the control information to the UE by DCI (downlink control information). DCI formats $1 \mathrm{~B}, 1 \mathrm{D}$ and 2 contain the information regarding the precoding vector/matrix employed by the eNodeB in the field TPMI (transmitted precoding matrix index). In these formats, 1 bit is reserved for the indication that the precoding is either according to the latest PMI report on PUSCH or is according to the indicated TPMI. Formats 1B and 1D concern the transmission of one PDSCH (physical downlink shared channel) codeword and have 2 bits in TPMI field to indicate one of the four precoders (once eNodeB has 2 antennas). Format 1D has 1 additional bit for the downlink power offset. DCI format 2 is more general and can be used for transmission mode 2, transmission mode 4, multi-user MIMO mode (transmission mode 5) and single-user MIMO mode (transmission mode 6). It therefore concerns the transmission of one or two PDSCH codewords and has 3 bits for the TPMI field. Though some bit fields are reserved in these DCI formats, but these formats neither contain the information regarding the precoder nor the constellation of the other coscheduled UE in the multi-user MIMO mode.

For the downlink transmission schemes at physical layer, a set of downlink reference signals have been defined. These reference signals can be either UE specific or cell specific. The cell specific signals are referred as common reference signals (CRS) while the UE specific signals are referred as dedicated (or demodulation) reference signals (DRS or DMRS). The CRS are used for channel estimation by the UEs so they are not precoded. The DM-RS are precoded and used for demodulation purposes on the scheduled physical resource blocks (PRB).

\section{B. System Model}

As our focus in this paper is on multi-user MIMO mode, so we consider the system model for transmission mode 5 . Transmitter block diagram is shown in Fig. 1. During the transmission for UE-1, the code sequence $\underline{\mathbf{c}}_{1}$ is interleaved by $\pi_{1}$ and then is mapped onto the signal sequence $\underline{\mathbf{x}}_{1}$. Bit interleaver for UE-1 can be modeled as $\pi_{1}: k^{\prime} \rightarrow(k, i)$ where $k^{\prime}$ denotes the original ordering of the coded bits $c_{k^{\prime}}, k$ denotes the resource element (RE - LTE acronym for subcarrier) of the symbol $x_{1, k}$ and $i$ indicates the position of the bit $c_{k^{\prime}}$ in the symbol $x_{1, k}$. Note that $x_{1, k} \in \chi_{1}$ is a symbol of $\underline{\mathbf{x}}_{1}$ where $\chi_{1} \subseteq \mathcal{C}$. Each RE corresponds to a symbol from a constellation map $\chi_{1}$ for UE-1 and $\chi_{2}$ for UE-2. Selection 


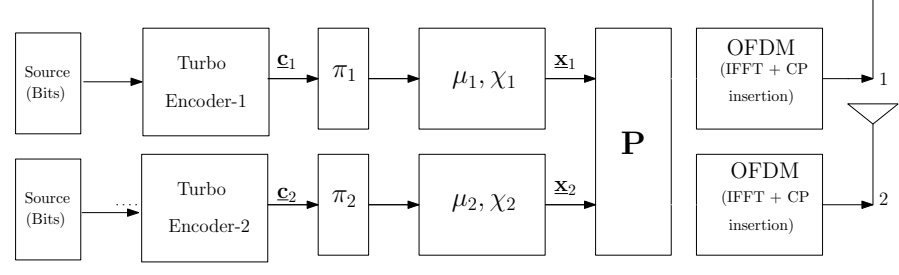

Fig. 1. eNodeB in multi-user MIMO mode. $\pi_{1}$ denotes the random interleaver, $\mu_{1}$ the labeling map and $\chi_{1}$ the signal set for the codeword of UE-1. $\mathbf{P}$ indicates precoding matrix.

of normal or extended cyclic prefix for each OFDM symbol converts downlink frequency selective channels into parallel flat fading channels. Cascading IFFT at the eNodeB and FFT at the UE with the cyclic prefix extension, transmission at the $k$-th RE for single antenna UE-1 in multi-user MIMO mode (transmission mode 5) can be expressed as

$$
y_{1, k}=\mathbf{h}_{1, k}^{\dagger} \mathbf{p}_{1, k} x_{1, k}+\mathbf{h}_{1, k}^{\dagger} \mathbf{p}_{2, k} x_{2, k}+z_{1, k}
$$

where $y_{1, k}$ is the received symbol at UE- 1 and $z_{1, k}$ is the zero mean circularly symmetric complex white Gaussian noise of variance $N_{0}$. Complex symbols $x_{1, k}$ and $x_{2, k}$ are assumed to be independent and of variances $\sigma_{1}^{2}$ and $\sigma_{2}^{2}$ respectively. $\mathbf{h}_{n, k}^{\dagger} \in \mathbb{C}^{1 \times 2}$ symbolizes the spatially uncorrelated flat Rayleigh fading MISO channel from the eNodeB to the $n$-th UE $(n=1,2)$ at the $k$-th RE. $\mathbb{C}^{1 \times 2}$ denotes the two dimensional complex space. $\mathbf{p}_{n, k}$ denotes the precoding vector for the $n$-th UE at the $k$-th RE and is given by (1). For the case of dual antenna UEs, the system equation is modified as

$$
\mathbf{y}_{1, k}=\mathbf{H}_{k}\left[\mathbf{p}_{1, k} x_{1, k}+\mathbf{p}_{2, k} x_{2, k}\right]+\mathbf{z}_{1, k}
$$

where $\mathbf{y}_{1, k}, \mathbf{z}_{1, k} \in \mathbb{C}^{2 \times 1}$ are the vectors of the received symbols and circularly symmetric complex white Gaussian noise of double-sided power spectral density $N_{0} / 2$ at 2 receive antennas of UE-1 while $\mathbf{H}_{k} \in \mathbb{C}^{2 \times 2}$ is the channel from eNodeB to UE-1.

\section{INTERFERENCE SENSITIVITY}

We first consider the case of single antenna UEs. As the processing at the UE is performed on a RE basis for each received OFDM symbol, the dependency on RE index can be ignored for notational convenience thereby leading to the system equation for transmission mode 5 as

$$
y_{1}=\mathbf{h}_{1}^{\dagger} \mathbf{p}_{1} x_{1}+\mathbf{h}_{1}^{\dagger} \mathbf{p}_{2} x_{2}+z_{1}
$$

Each UE feeds back the precoder which maximizes its received signal strength which would obviously be a matched filter precoder. Based on its channel estimation by CRS, the UEs compute the quantized versions of their MF precoders, i.e. if channel of the UE from eNodeB is $\mathbf{h}_{1}^{\dagger}=\left[\begin{array}{ll}h_{11}^{*} & h_{21}^{*}\end{array}\right]$, it first computes its MF precoder $\left[\begin{array}{ll}h_{11} & h_{21}\end{array}\right]^{T}$ followed by searching the precoder in the LTE codebook which is closest to the MF precoder. This can be done by either calculating the Euclidean distance between the MF precoder and LTE precoders or can also be done by computing the angle from $h_{11}^{*}$ to $h_{21}^{*}$ i.e. $\angle\left(h_{11} h_{21}^{*}\right)$, and looking for the LTE precoder whose phase $\left[0^{\circ}, \pm 90^{\circ}, 180^{\circ}\right]$ is closest to the conjugate of $\angle\left(h_{11} h_{21}^{*}\right)$. For multi-user MIMO mode, the eNodeB selects two UEs to be served on the same physical resources which have good channel separation i.e. the UEs which have fed back opposite precoders, i.e. the eNodeB selects as the second UE one of the UEs whose requested precoder $\mathbf{p}_{2}$ is $180^{\circ}$ out of phase from the precoder $\mathbf{p}_{1}$ of the first UE to be served on the same physical resources. The precoder matrix is therefore given as $\mathbf{P}=\frac{1}{\sqrt{2}}\left[\begin{array}{cc}1 & 1 \\ q & -q\end{array}\right]$ where $q=[ \pm 1 ; \pm j]$. So the signal received by $\mathrm{UE}-1$ is given as

$$
y_{1}=\frac{1}{\sqrt{2}}\left(h_{11}^{*}+q h_{21}^{*}\right) x_{1}+\frac{1}{\sqrt{2}}\left(h_{11}^{*}-q h_{21}^{*}\right) x_{2}+z_{1}
$$

Interference aware receiver structure [10] for multi-user MIMO is based on Max log MAP detection. Based on this detection rule, the bit metric for bit $c_{k^{\prime}}$ of $x_{1}$ is given as [8]

$$
\Lambda_{1}^{i}\left(y_{1}, c_{k^{\prime}}\right) \approx \min _{x_{1} \in \chi_{1, c_{k^{\prime}}}^{i}, x_{2} \in \chi_{2}}\left|y_{1}-\mathbf{h}_{1}^{\dagger} \mathbf{p}_{1} x_{1}-\mathbf{h}_{1}^{\dagger} \mathbf{p}_{2} x_{2}\right|^{2}
$$

where $\chi_{1, c_{k^{\prime}}}^{i}$ denotes the subset of the signal set $x_{1} \in \chi_{1}$ whose labels have the value $c_{k^{\prime}} \in\{0,1\}$ in the position $i$. The employment of this bit metric necessitates the information regarding the precoder of the interference $\left(\mathbf{p}_{2}\right)$ and the constellation of the interference $\left(\chi_{2}\right)$. The scheduling strategy enables UE-1 to find $\mathbf{p}_{2}$ being $180^{\circ}$ out of phase of $\mathbf{p}_{1}$ but it lacks the information of $\chi_{2}$. Here we propose blind receiver structure in which the interference is always assumed to be QAM16. As LTE supports three constellations as QPSK, QAM16 and QAM64, so this postulation will not only capture the effect of four quadrants of QPSK constellation points but will also encapsulate the spread of QAM64 constellation points in each quadrant thereby leading to a reasonable compromise.

Based on [10], we develop the bit metric further by writing terms in their real and imaginary parts

$$
\begin{gathered}
\Lambda_{1}^{i}\left(y_{1}, c_{k^{\prime}}\right) \approx \min _{x_{1} \in \chi_{1, k_{k^{\prime}}}^{i}, x_{2} \in \chi_{2}}\left\{\left|y_{1}\right|^{2}+\left|\mathbf{h}_{1}^{\dagger} \mathbf{p}_{1} x_{1}\right|^{2}-2\left(\mathbf{h}_{1}^{\dagger} \mathbf{p}_{1} x_{1} y_{1}^{*}\right)_{R}\right. \\
\left.+2\left(\rho_{12} x_{1}^{*} x_{2}\right)_{R}-2\left(\mathbf{h}_{1}^{\dagger} \mathbf{p}_{2} x_{2} y_{1}^{*}\right)_{R}+\left|\mathbf{h}_{1}^{\dagger} \mathbf{p}_{2} x_{2}\right|^{2}\right\} \\
\approx \min _{x_{1} \in \chi_{1, c_{k^{\prime}}^{\prime}, x_{2} \in \chi_{2}}^{i}}\left\{\left|\mathbf{h}_{1}^{\dagger} \mathbf{p}_{1} x_{1}\right|^{2}-2\left(\bar{y}_{1}^{*} x_{1}\right)_{R}\right. \\
\left.+2\left(\psi_{A}\right) x_{2, R}+\left|\mathbf{h}_{1}^{\dagger} \mathbf{p}_{2}\right|^{2} x_{2, R}^{2}+2\left(\psi_{B}\right) x_{2, I}+\left|\mathbf{h}_{1}^{\dagger} \mathbf{p}_{2}\right|^{2} x_{2, I}^{2}\right\}
\end{gathered}
$$

where the subscript $(.)_{R}$ indicates the real part and the subscript (.) $)_{I}$ indicates the imaginary part. $\rho_{12}=\left(\mathbf{h}_{1}^{\dagger} \mathbf{p}_{1}\right)^{*} \mathbf{h}_{1}^{\dagger} \mathbf{p}_{2}$ indicates the cross correlation between the two coefficients while $\bar{y}_{1}=\left(\mathbf{h}_{1}^{\dagger} \mathbf{p}_{1}\right)^{*} y_{1}$ and $\bar{y}_{2}=\left(\mathbf{h}_{1}^{\dagger} \mathbf{p}_{2}\right)^{*} y_{1}$ indicate the 
outputs of the MF. Note that

$$
\begin{aligned}
& \psi_{A}=\rho_{12, R} x_{1, R}+\rho_{12, I} x_{1, I}-\bar{y}_{2, R} \\
& \psi_{B}=\rho_{12, R} x_{1, I}-\rho_{12, I} x_{1, R}-\bar{y}_{2, I}
\end{aligned}
$$

Focusing on the signs of $x_{2, R}$ and $x_{2, I}$, they should be in the opposite directions of $\psi_{A}$ and $\psi_{B}$ respectively. So the bit metric is written as

$$
\begin{aligned}
& \Lambda_{1}^{i}\left(y_{1}, c_{k^{\prime}}\right) \approx \min _{x_{1} \in \chi_{1, c_{k^{\prime}}^{i}, x_{2} \in \chi_{2}}}\left\{\left|\mathbf{h}_{1}^{\dagger} \mathbf{p}_{1} x_{1}\right|^{2}-2\left(\bar{y}_{1}^{*} x_{1}\right)_{R}\right. \\
& \left.-2\left|\psi_{A}\right|\left|x_{2, R}\right|+\left|\mathbf{h}_{1}^{\dagger} \mathbf{p}_{2}\right|^{2}\left|x_{2, R}\right|^{2}-2\left|\psi_{B}\right|\left|x_{2, I}\right|+\left|\mathbf{h}_{1}^{\dagger} \mathbf{p}_{2}\right|^{2}\left|x_{2, I}\right|^{2}\right\}
\end{aligned}
$$

Regarding the magnitudes of $x_{2, R}$ and $x_{2, I}$ which minimize (4), it is the minimization problem of a quadratic function, i.e. differentiating (4) w.r.t $\left|x_{2, R}\right|$ and $\left|x_{2, I}\right|$ to find the global minimas which are given as

$$
\left|x_{2, R}\right|=\frac{\left|\psi_{A}\right|}{\left|\mathbf{h}_{1}^{\dagger} \mathbf{p}_{2}\right|^{2}}, \quad\left|x_{2, I}\right|=\frac{\left|\psi_{B}\right|}{\left|\mathbf{h}_{1}^{\dagger} \mathbf{p}_{2}\right|^{2}}
$$

As the values of $x_{2, R}$ and $x_{2, I}$ for the case of QAM16 are $\left[ \pm \frac{\sigma_{2}}{\sqrt{10}}, \pm \frac{3 \sigma_{2}}{\sqrt{10}}\right]$ so their magnitudes in (4) are given as

$$
\begin{aligned}
& \left|x_{2, R}\right|=\sigma_{2} \frac{1}{\sqrt{10}}\left(2+(-1)^{I\left(\left|\psi_{A}\right|<\sigma_{2} \frac{2\left|\mathbf{h}_{1}^{\dagger} \mathbf{p}_{2}\right|^{2}}{\sqrt{10}}\right)}\right) \\
& \left|x_{2, I}\right|=\sigma_{2} \frac{1}{\sqrt{10}}\left(2+(-1)^{I\left(\left|\psi_{B}\right|<\sigma_{2} \frac{2\left|\mathbf{h}_{1}^{\dagger} \mathbf{p}_{2}\right|^{2}}{\sqrt{10}}\right)}\right)
\end{aligned}
$$

and $I($.$) is the indicator function defined as$

$$
I(a<b)= \begin{cases}1 & \text { if } a<b \\ 0 & \text { otherwise }\end{cases}
$$

The bit metric (4) is calculated based on the values of $x_{2, R}$ and $x_{2, I}$ from (6) and (7). The detection is therefore independent of the constellation information of the interference $x_{2}$. The derivation of this metric remains same for the case of dual antenna UEs where some terms are redefined as $\bar{y}_{1}=\left(\mathbf{H p}_{1}\right)^{\dagger} \mathbf{y}$, $\bar{y}_{2}=\left(\mathbf{H} \mathbf{p}_{2}\right)^{\dagger} \mathbf{y}$ and $p_{12}=\left(\mathbf{H} \mathbf{p}_{1}\right)^{\dagger} \mathbf{H} \mathbf{p}_{2}$. Moreover $\mathbf{h}_{1}^{\dagger} \mathbf{p}_{1}$ and $\mathbf{h}_{1}^{\dagger} \mathbf{p}_{2}$ are replaced by $\mathbf{H} \mathbf{p}_{1}$ and $\mathbf{H} \mathbf{p}_{2}$ respectively.

\section{Simulations}

For simulations, we consider the downlink of 3GPP LTE (BICM OFDM transmission) with the eNodeB equipped with two antennas using rate-1/3 LTE turbo code [7] punctured to rate $1 / 2^{1}$. We focus on LTE multi-user MIMO mode and consider dual antenna UEs. We consider ideal OFDM system (no ISI) and analyze the system in the frequency domain where the channel has iid Gaussian matrix entries with unit variance and is independently generated for each channel use. We assume no power control in multi-user MIMO mode so

\footnotetext{
${ }^{1}$ The LTE turbo decoder design was performed using the coded modulation library www.iterativesolutions.com
}

two UEs have equal power distribution. It is assumed that UE knows its own channel from the eNodeB, so UE can find the effective channel of the co-scheduled UE (interference) based on the fact that the eNodeB schedules the second UE on the same RE which has requested $180^{\circ}$ out of phase precoder with reference to the precoder of the first UE. We focus on the frame error rates (FER) while the frame length is fixed to 1056 information bits.

Fig. 2 compares the FER of UE-1 for the cases when the constellation of the co-scheduled UE-2 is not known by UE1. In this scenario, UE-1 employs the proposed blind receiver structure based on assuming interference to be QAM16. For comparison purposes, we also consider the cases when UE1 assumes unknown interfering constellation to be QPSK, QAM64, QAM256 and Gaussian (single-user receiver). These results show that there is negligible degradation in the performance of the multi-user MIMO mode if the interfering constellation is assumed to be from higher order modulation. However, there is significant degradation if interference is assumed to be from lower order modulation. It is attributed to the fact that assuming interference to be from a higher order modulation amongst the possible modulation alphabets leads to the best compromise as this assumption includes the lower modulation orders as special cases (with proper scaling). As LTE and LTE-Advanced restrict the transmission to three modulations as QPSK, QAM16 and QAM64, so assuming interference to be QAM64 (or even QAM16) leads to better performance. Considering the complexity and performance, assuming interference to be QAM16 is the best compromise in LTE which is the case for our proposed blind receiver. Assuming interference to be Gaussian thereby leading to single-user detection leads to degraded performance especially at higher spectral efficiencies. Note that the case of QAM256 has been included for better understanding of the behavior otherwise both LTE and LTE-Advanced do not support this modulation. These results show that exploiting the discrete nature of interference even when its constellation is not known leads to much improved performance as compared to the case of assuming this unknown interference to be Gaussian.

\section{CONCLUSION}

In this paper we have looked at the sensitivity of multiuser MIMO mode in LTE to the interfering constellation. This sensitivity analysis is motivated by the fact that DCI formats in LTE multi-user MIMO mode do not include the information of the constellation of co-scheduled UE. We have shown that exploiting the discrete nature of interference even if its exact constellation is not known leads to much improved performance as compared to its Gaussian assumption. Based on this, we have proposed a blind receiver structure for multiuser MIMO mode in LTE. However the possible gains with the efficient exploitation of interference structure motivates the inclusion of the information of the constellation of co-scheduled UE in the ongoing standardizations of LTE-Advanced. 

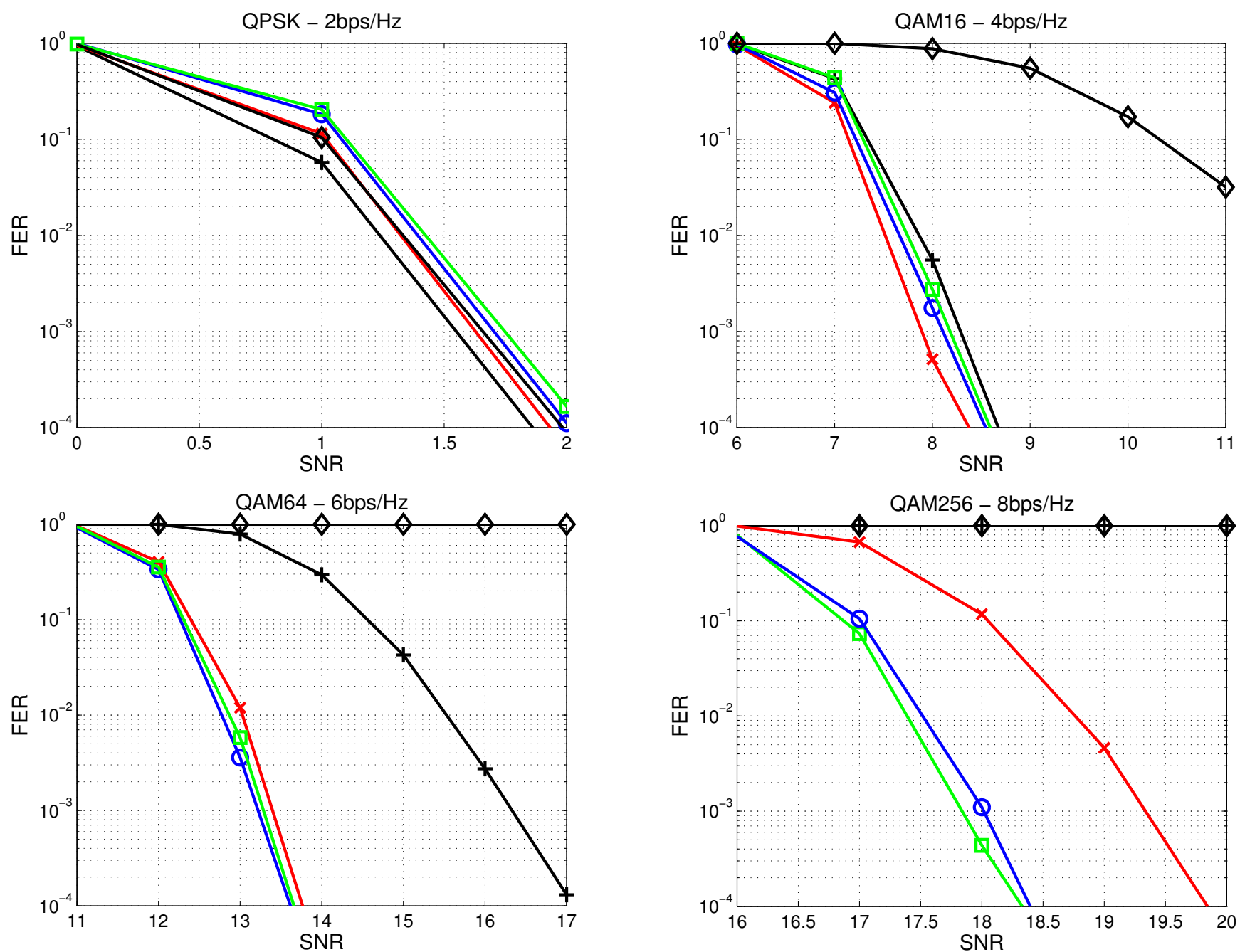

\begin{tabular}{|c|c|c|c|c|c|c|c|}
\hline+ & $\begin{array}{l}\text { Interference } \\
\text { assumed to be } \\
\text { QPSK }\end{array}$ & $\begin{array}{c}\text { Interference } \\
\text { assumed to be } \\
\text { QAM16 }\end{array}$ & $\theta$ & $\begin{array}{c}\text { Interference } \\
\text { assumed to be } \\
\text { QAM64 }\end{array}$ & $\square$ & $\begin{array}{l}\text { Interference } \\
\text { assumed to be } \\
\text { QAM256 }\end{array}$ & $\widehat{\nabla} \stackrel{\text { Single-user }}{\text { detection }}$ \\
\hline
\end{tabular}

Fig. 2. Interference sensitivity for multi-user MIMO mode in LTE. Four sets of simulations are shown. QPSK indicates that both $x_{1}$ and $x_{2}$ belong to QPSK.

\section{ACKNOWLEDGMENTS}

Eurecom's research is partially supported by its industrial partners: BMW, Bouygues Telecom, Cisco Systems, France Télécom, Hitachi Europe, SFR, Sharp, ST Microelectronics, Swisscom, Thales. The research work leading to this paper has also been partially supported by the European Commission under SAMURAI and IST FP7 research network of excellence NEWCOM++.

\section{REFERENCES}

[1] I. E. Telatar, "Capacity of multiantenna Gaussian channels," European Transactions on Telecommunications, vol. 10, no. 6, pp. 585-595, Nov./Dec 1999.

[2] LTE, Evolved Universal Terrestrial Radio Access (E-UTRA); Physical Layer Procedures, Release 8, V.8.6.0. 3GPP TS 36.213, 2009.

[3] LTE-A, Requirements for Further Advancements for Evolved Universal Terrestrial Radio Access (EUTRA) (LTE-Advanced). 3GPP TR 36.913, 2008.
[4] D. Love, R. Heath, V. Lau, D. Gesbert, B. Rao, and M. Andrews, "An overview of limited feedback in wireless communication systems," IEEE Journal on Selected Areas in Communications, vol. 26, no. 8, pp. 1341 -1365 , Oct. 2008

[5] S. Sesia, I. Toufik, and M. Baker, LTE, The UMTS Long Term Evolution: From Theory to Practice. Wiley, 2009.

[6] R. Ghaffar and R. Knopp, "Making Multiuser MIMO work for LTE," in IEEE 21-st International Symposium on Personal, Indoor and Mobile Radio Communications (PIMRC 2010), Istanbul, September 2010.

[7] LTE, Evolved Universal Terrestrial Radio Access (E-UTRA); Channel Coding and Multiplexing, Release 8, V.8.6.0. 3GPP TS 36.212, 2009.

[8] G. Caire, G. Taricco, and E. Biglieri, "Bit-interleaved coded modulation," IEEE Transactions on Information Theory, vol. 44, no. 3, pp. 927-946, May 1998.

[9] S. Alamouti, "A simple transmit diversity technique for wireless communications," IEEE Journal on Selected Areas in Communications, vol. 16, no. 8, pp. 1451-1458, Oct 1998.

[10] R. Ghaffar and R. Knopp, "Linear precoders for multiuser MIMO for finite constellations and a simplified receiver structure under controlled interference," in Asilomar Conference on Signals, Systems and Computers, Nov. 2009. 